Check for updates

Cite this: RSC Adv., 2017, 7, 56153

7th October 2017

Accepted 7th November 2017

DOI: $10.1039 / \mathrm{c} 7 \mathrm{ra11033b}$

rsc.li/rsc-advances

\section{Relaxor-like and switchable dielectric behavior in a rare noncentrosymmetric 3D iodoargentate hybrid $\dagger$}

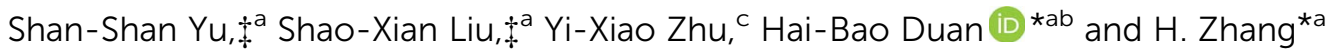

 \\ prepared using a simple solution process. 1 crystallized in the hexagonal space group $P 6_{3} 22$. Its \\ inorganic components formed a novel 3D open-framework structure. 1 is the first 3D iodoargentate \\ hybrid shown to have relaxor-like dielectric behavior and switchable dielectric properties.
}

Hybrid iodoargentates have been intensively investigated not only because of their flexible coordination modes and bonding arrangements, ${ }^{1,2}$ but also because of their novel physical properties applicable in fields from optics ${ }^{3}$ to electronics. ${ }^{4}$ Many anionic iodoargentates with the general formula $\left(\mathrm{Ag}_{m} \mathrm{I}_{n}\right)^{(n-m)-}$ have been synthesized and structurally characterized, such as zero-dimensional (OD) clusters of $\left[\mathrm{Ag}_{4} \mathrm{I}_{8}\right]^{4-},\left[\mathrm{Ag}_{5} \mathrm{I}_{9}\right]^{4-},\left[\mathrm{Ag}_{5} \mathrm{I}_{10}\right]^{5-}$, $\left[\mathrm{Ag}_{6} \mathrm{I}_{12}\right]^{6-}$ and $\left[\mathrm{Ag}_{7} \mathrm{I}_{13}\right]^{6-5,6}$ one-dimensional (1D) chains of $\left[\mathrm{AgI}_{2}\right]^{-},\left[\mathrm{Ag}_{2} \mathrm{I}_{5}\right]^{3-},\left[\mathrm{Ag}_{4} \mathrm{I}_{6}\right]^{2-},\left[\mathrm{Ag}_{5} \mathrm{I}_{7}\right]^{2-}$ and $\left[\mathrm{Ag}_{6} \mathrm{I}_{8}\right]^{2-}, 7,8$ twodimensional (2D) $\mathrm{S}$ of $\left[\mathrm{Ag}_{2} \mathrm{I}_{3}\right]^{-}, \quad\left[\mathrm{Ag}_{4} \mathrm{I}_{5}\right]^{-}, \quad\left[\mathrm{Ag}_{5} \mathrm{I}_{6}\right]^{-}$and $\left[\mathrm{Ag}_{11} \mathrm{I}_{15}\right]^{4-}$, ${ }^{9}$ and three-dimensional (3D) frameworks of $\left[\mathrm{AgI}_{2}\right]^{-}$, $\left[\mathrm{Ag}_{4} \mathrm{I}_{6}\right]^{-}$and $\left[\mathrm{Ag}_{5} \mathrm{I}_{7}\right]^{2-}$. $^{a}$ Three-dimensional iodoargentates are non-oxide analogues of microporous zeolites and have led to new developments in host-guest chemistry through crystal engineering of the building blocks of their chemical frameworks, but there are few reported examples of 3D iodoargentates. Noncentrosymmetric 3D iodoargentates are of great importance not only due to their interesting structural features but also because they have been exploited in second-order nonlinear, ferroelectric, and other related applications. However, it is still challenging to synthesize noncentrosymmetric 3D iodoargentates, especially to effectively utilize agents that direct the formation of achiral structures. ${ }^{10}$

On the other hand, supramolecular chemistry and molecular crystal engineering, which involve the planning

\footnotetext{
${ }^{a}$ Key Laboratory of Advanced Functional Materials of Nanjing, Nanjing Xiaozhuang University, Nanjing 211171, P. R. China. E-mail: duanhaibao4660@163.com

${ }^{b}$ Department of Chemistry and Biochemistry, University of California, Los Angeles, California 90095-1569, USA

'Nanjing Foreign Language School, Nanjing 210018, P. R. China

$\dagger$ Electronic supplementary information (ESI) available: Experimental details, crystal data, additional figures, power XRD, TG, dielectric and conductivity curves. CCDC 1559713. For ESI and crystallographic data in CIF or other electronic format see DOI: $10.1039 / \mathrm{c} 7 \mathrm{ra11033b}$

‡ Shan-Shan Yu and Shao-Xian Liu contribute equally.
}

and utilization of crystal-oriented syntheses for the bottomup construction of functional molecular solids from molecules and ions, are powerful tools that have been used for the assembly of designed functional materials over the past three decades. ${ }^{11}$ In our previous study, we focused our research on the haloplumbate hybrids, and some soft organic components were introduced into haloplumbate crystal lattices. Those haloplumbate hybrid compounds showed arresting ferroelectric, dielectric and thermochromic luminescence properties. ${ }^{12-14}$ Recent investigation showed localized charged states to be strongly coupled to local structural lattice distortions and cation rotations in haloplumbate hybrid crystals $^{15}$ and to induce dielectric relaxation behavior. Compared to the $\mathrm{Pb}^{2+}$ hybrid, $\mathrm{Ag}^{1}$ with a d ${ }^{10}$ closedshell electronic configuration has shown flexible arrangements around the metal center and resulting local polar fluctuations have been shown to result in interesting photoelectric properties. Here, we obtained a rare 3D iodoargentate hybrid, namely, $\left[\mathrm{Cd}(\mathrm{en})_{3}\right] \mathrm{Ag}_{2} \mathrm{I}_{4}$ (1), and characterized its dielectric properties. A frequency-dependent dielectric dispersion phenomenon was observed above room temperature and analyzed using impedance spectroscopy. The dielectric relaxation behavior of $\mathbf{1}$ may have been due to local polarization effects of the framework. 1 is the first 3D iodoargentate hybrid shown to have dielectric relaxation and switchable dielectric behaviors.

The compound 1 crystal was synthesized by combining a DMF solution of $\mathrm{Cd}\left(\mathrm{NO}_{3}\right)_{2}$ and $2 \mathrm{ml}$ of an ethylenediamine and $\mathrm{KAgI}_{2} / \mathrm{DMF}$ solution. The resulting solution was kept at room temperature for two weeks, and white block crystals were formed in $c a$. $90 \%$ yield based on CD (see ESI $\dagger$ ). The phase purity of the as-prepared sample was characterized by carrying out powder X-ray diffraction (PXRD), elemental analyses of C, $\mathrm{H}$ and N, and IR spectroscopy (Fig. S1†). TG was used to analyze its stability up to $300{ }^{\circ} \mathrm{C}$ (Fig. S2 $\dagger$ ). The second-order nonlinear optical properties for $\mathbf{1}$ were examined, and indicated that the 
powdered samples of 1 were SHG active with a response $c a$. 0.5 times that of urea. $\S$

Compound 1 crystallized in the hexagonal space group $P 6_{3} 22$ (no. 182), which belongs to the chiral point group $D_{6}$, and with an asymmetric unit containing two crystallographically distinct $\mathrm{Ag}^{+}$ions and two crystallographically distinct $\mathrm{I}^{-}$ions together with one dication complex (as depicted in Fig. 1a). The inorganic part of $\mathbf{1}$ formed a novel 3D open-framework structure, made up of tetrahedral $\mathrm{AgI}_{4}$ building blocks. The Ag-I bond length was measured to be in the range 2.882-2.892 $\AA$, all within the van der Waals contact limit. The Ag-I-Ag bond angles ranged from 104.7 to $113.7^{\circ}$, slightly deviating from $109.5^{\circ}$ for an ideal tetrahedron. All of the $\mathrm{I}^{-}$anions formed $\mu_{2}$ bridges connecting two neighboring $\mathrm{Ag}^{+}$ions. Thus, each $\mathrm{Ag}_{1} \mathrm{I}_{4}$ tetrahedron shared its vertex with another four different $\mathrm{Ag}_{2} \mathrm{I}_{4}$ tetrahedra (Fig. 1b). Six AgI $\mathrm{I}_{4}$ tetrahedra were observed to stack into a 12-ring structure (Fig. 1c), and a non-interpenetrating tridymite-type topology formed from the 12-ring structure. Along the $a$ - and $b$-axis directions, an approximately rectangular inorganic channel was observed and measured to have dimensions of about $5.77 \times 9.25 \AA$ without considering internal van der Waals radii (Fig. 1d and e). Furthermore, another irregular 12-ring channel formed along the $c$ axis (Fig. 1f). The channels along the three directions were observed to merge into a $3 \mathrm{D}$ channel network, a rare example of an open silver halide framework, and $\mathrm{Cd}(\mathrm{en})_{3}{ }^{2+}$ dications filled the channels (Fig. 1e). The $\mathrm{Cd}^{2+}$ ions of the $\mathrm{Cd}(\mathrm{en})_{3}{ }^{2+}$ dications were observed to occupy the Wyckoff position, its coordination octahedron, with a $\mathrm{C}_{3}$ point group symmetry, was built from six nitrogen atoms from three ethylenediamine molecule. Charged H-bonding interactions formed between the dications and 3D inorganic framework.

Dielectric relaxation investigation were carried out for temperatures between 50 and $140{ }^{\circ} \mathrm{C}$ using the complex modulus (Fig. 2 and $\mathrm{S} 4 \dagger$ ). The electric modulus $\left(M^{*}\right)$ was calculated according to the equation

$$
M^{*}(\omega)=\frac{1}{\varepsilon^{*}(\omega)}=\frac{\varepsilon^{\prime}+\mathrm{j} \varepsilon^{\prime \prime}}{\varepsilon^{\prime 2}+\varepsilon^{\prime \prime 2}}=M^{\prime}+\mathrm{j} M^{\prime \prime},
$$

where $M^{\prime}$ and $M^{\prime \prime}$ are the real and imaginary parts of the complex modulus $M^{*}$, respectively. At low frequencies, $M^{\prime}$ was calculated to be very small, to indicative of negligible contributions of the electrode polarization and space charge injection effects. The $M^{\prime}$ showed a tendency to saturate at high frequencies, which is typical for dielectric relaxation process in the investigated temperature range (Fig. 2a). The shift of the $M^{\prime}$ maximum slightly toward a higher temperature upon

$\S$ Crystal data for compound 1 at $293 \mathrm{~K}_{2} \mathrm{C}_{6} \mathrm{H}_{24} \mathrm{Ag}_{2} \mathrm{CdI}_{4} \mathrm{~N}_{6}, M=1016.06$, hexagonal, space group $P 6_{3} 22, a=9.2512(12) \AA ̊ ., b=9.2512(12) \AA, c=14.504(5) \AA, \alpha=\beta=\gamma=$ $90.00^{\circ}, V=1075.0(5) \AA^{3}, Z=2, D_{\mathrm{c}}=3.139 \mathrm{~g} \mathrm{~cm}^{-3} . \mu=8.525 \mathrm{~mm}^{-1} ; F(000)=912.0$, $R_{1}=0.0468, \mathrm{w} R_{2}=0.1050$ for 1002 unique reflections and 37 parameters, $S=$ 1.228, CCDC-1559713. Single-crystal diffraction data for 1 were collected on a Siemens SMART-CCD diffractometer with graphite monochromatic Mo K $\alpha$ radiation $(\lambda=0.71073 \AA)$. The structures were solved using direct methods and refined on $F^{2}$ using a full matrix least-squares method with SHELXTL. Anisotropic thermal parameters were refined for the non-hydrogen atoms. All $\mathrm{H}$ atoms were placed at calculated positions and refined riding on the parent atoms. (a)

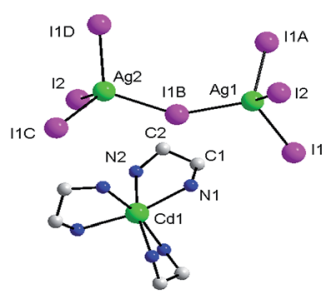

(c)

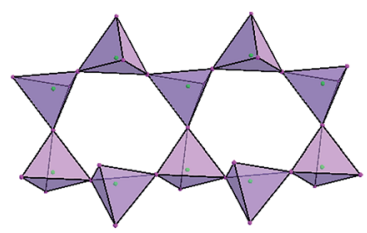

(e)

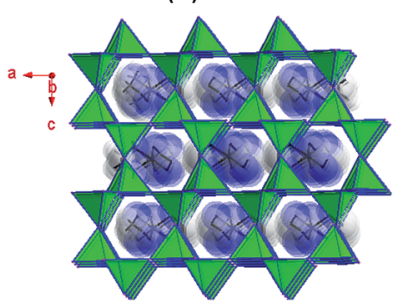

(b)

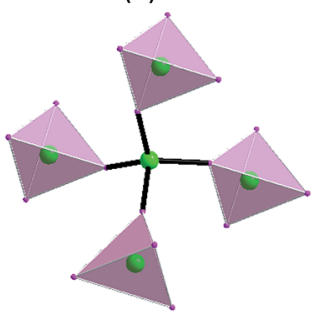

(d)

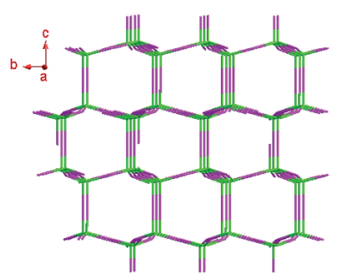

(f)

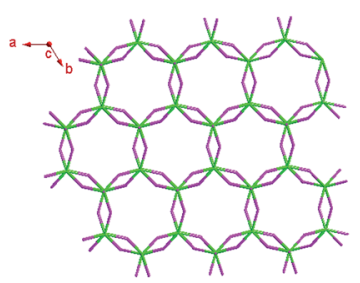

Fig. 1 (a) The asymmetric unit of 1 at $293 \mathrm{~K}$ with $50 \%$ thermal ellipsoid probability. (b) Each $\mathrm{Agl}_{4}$ tetrahedron was observed to share its vertex with another four different $\mathrm{Agl}_{4}$ tetrahedra. (c) 12-ring structure formed by six $\mathrm{Agl}_{4}$ tetrahedra. (d) View of an approximately rectangular inorganic channel along the $a$-axis direction. Dications in the channel are omitted for clarity. (e) Packing structure along the $b$-axis, with $\mathrm{Cd}(\mathrm{en})_{3}{ }^{2+}$ dications filling the channels and shown in space-filling mode. (f) Another irregular channel along the $c$-axis. (Symmetry relationships: $A=-x, 1-y,-z ; B=1-x, 1-y, 1+z ; C=1-x, 1+y, z ; D=$ $x,-y, 0.5-z$.)

increasing the frequency of the applied electrical field further indicated a thermally activated process. Furthermore, the contribution of conduction to frequency was due to the shortrange mobility of the charge carriers. In the $M^{\prime \prime}-f$ plots, the spectrum at the investigated temperature exhibited a relaxation peak (Fig. 2b). The peaks shifted systematically towards higher frequencies as the temperature was increased. This shift implied a redevelopment of the polarization at high temperature, and thus the occurrence of relaxation at high frequency. In order to gain deep insight into the dielectric relaxation process, the frequency dependence of the peak for $M^{\prime \prime}$ at different temperatures was plotted (Fig. 2c) according to the relationship

$$
\tau=\tau_{0} \exp \left(\frac{E_{\mathrm{a}}}{k_{\mathrm{B}} T}\right) .
$$

In this equation, $\tau=1 / f_{\max }$, where $f_{\max }$ is the frequency at the maximum of $M^{\prime \prime}-f$ in the plot at temperature $T, \tau_{0}$ represents the characteristic macroscopic relaxation time, $E_{\mathrm{a}}$ is the activation energy or potential barrier required for the dielectric relaxation, 
(a)

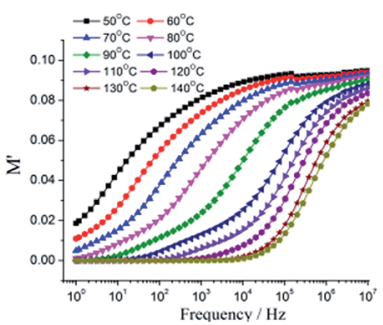

(c)

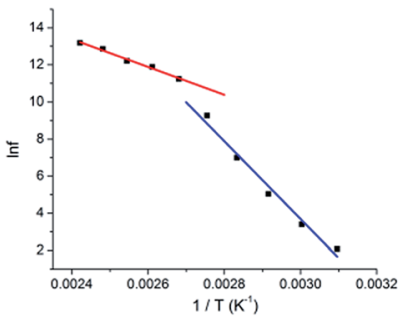

(b)

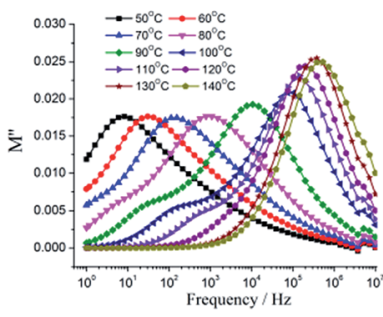

(d)

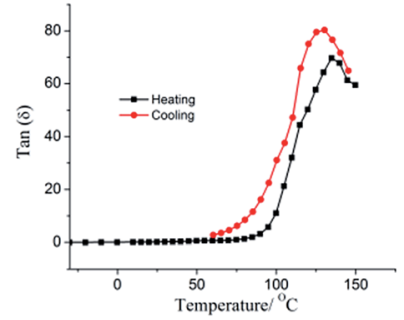

Fig. 2 ( $a$ and b) Frequency dependencies of the $M^{\prime}$ and $M^{\prime \prime}$ of 1 in the $50-140{ }^{\circ} \mathrm{C}$ temperature range, respectively. (c) Plots of $\ln f v s .1 / T$ for the relaxation at selected temperatures and $(d) \tan (\delta)$ versus temperature at $100 \mathrm{~Hz}$ for 1.

and $k_{\mathrm{B}}$ is Boltzmann's constant. Two steps of dielectric relaxation were found, as shown in Fig. $2 \mathrm{c}$. We found $\ln f_{\max }$ to be linearly related to $1 / T$ in the temperature ranges $323-363 \mathrm{~K}$ and $373-413 \mathrm{~K}$. The best fits gave the following results: $\tau_{0}=1.356 \times$ $10^{-29} \mathrm{~s}$ and $E_{\mathrm{a}}=21.07 \mathrm{kcal} \mathrm{mol}^{-1}$ in the temperature range $323-$ $363 \mathrm{~K}$ and $\tau_{0}=2.15 \times 10^{-14} \mathrm{~s}$ and $E_{\mathrm{a}}=7.53 \mathrm{kcal} \mathrm{mol}^{-1}$ in the temperature range $373-413 \mathrm{~K}$. The dielectric relaxation process was also indicated from the $\tan (\delta)-f$ plot $\left(\tan (\delta)=\varepsilon^{\prime \prime} / \varepsilon^{\prime}\right)$ (Fig. S3†), which showed a broad maximum at the selected temperature, and the maximum $\tan (\delta)$ peak shifted toward higher frequencies as the temperature was increased, indicating the occurrence of thermally active dielectric relaxation. However, the frequency dependence of $\tan (\delta)$ showed a strong low-frequency dispersion when the temperature was increased (Fig. S3 $\dagger$ ). When the temperature is above $100{ }^{\circ} \mathrm{C}$, the dielectric loss shows abrupt increase, which reaches the values of 6.31 for 1 and $10 \mathrm{~Hz}$. The temperature-dependent dielectric loss is shown in Fig. 2d. When the frequency was lower than $100 \mathrm{~Hz}$, an obvious dielectric anomaly was present at about $135{ }^{\circ} \mathrm{C}$ in the heating process. As the temperature was further increased, the $\tan (\delta)$ value decreased. During the subsequent cooling process, a small thermal hysteresis was obtained. The dielectric loss switching between high and low dielectric states and the thermal hysteresis indicated compound $\mathbf{1}$ to be a potential switchable bistable dielectric material.

In general, four different mechanisms for dielectric relaxation have been proposed. The electronic polarization and molecular vibration occurred at frequencies above $10^{12} \mathrm{~Hz}$. At the frequency range of $10^{6}-10^{12} \mathrm{~Hz}$, the dielectric relaxation was more prevalent than dipole motion or ionic polarization. Dielectric relaxation is usually found in disordered solids such as relaxor ferroelectrics, dipolar glass and inorganic-organic

hybrids, and can be ascribed to order-disorder phase transition; some metal-organic frameworks and ionic cocrystals also show dielectric relaxation behavior. ${ }^{\mathbf{1 6}}$ However, we were surprised to observe relaxor-like behavior in compound $\mathbf{1}$, which was shown to not have a disordered component; and the DSC results (Fig. S5 $\dagger$ ) indicated the absence of a phase transition in the investigated temperature range. In compound $\mathbf{1}, \mathrm{Ag}$ and I ions were observed to have large ion displacements, the 3D channel structure formed by compound $\mathbf{1}$ became more distorted as the temperature was increased. Thus, we suggested the dielectric relaxation behavior of $\mathbf{1}$ to be associated with local polarization effects due to the flexibility of the channel structure and, in particular, due to small local movements of the $\mathrm{Ag}$ and I ions in the framework to create local dipole moments.

The abrupt increase in dielectric loss at $100 \mathrm{~Hz}$ above $100{ }^{\circ} \mathrm{C}$. The dielectric loss reached a value of 10.25 at $105^{\circ} \mathrm{C}$, was due to the electronic conductance. ${ }^{17}$ To further investigate the dielectric and conduction behaviors of compounds 1 , the complex impedance $\left(Z^{\prime}-Z^{\prime \prime}\right)$ at selected temperatures was plotted (Fig. 3a and b). The semicircles were observed for the selected temperature. The low-frequency dispersion corresponded to the grain boundary and the high-frequency region corresponded to the grain interior. This observation indicated that a single conductivity process took place in the sample at the investigated frequency. The impedance spectra from Fig. 3a and b were interpreted by means of an equivalent circuit. Here, each impedance semicircle represents the total dc resistance of the sample, $R$, furthermore, bulk capacitor, $C$ in parallel (parallel $R C$ element) and $C_{\mathrm{x}}$ (Cole-Cole branch) represents polarization associated with the lattice relaxation process. The centers of these semicircles were located below the real axis, indicating a non-Debye type of relaxation process. The best fit to the data

(a)

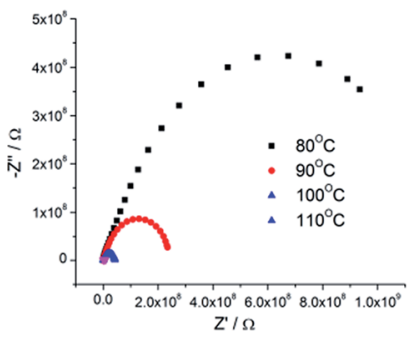

(c)

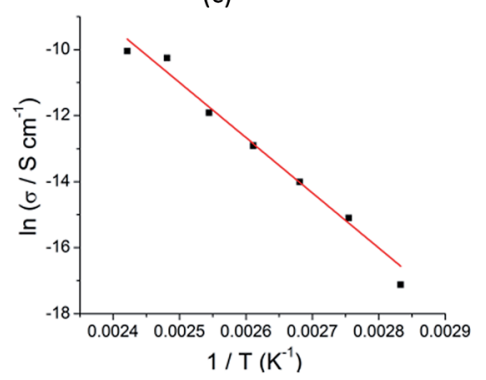

Fig. 3 (a and b) Complex-plane impedance plots at various temperatures. (c) Temperature dependence of conductivity for 1 (black dot: obtained from using an equivalent circuit; red line: theoretically reproduced using the Arrhenius equation). 
gave $\sigma=3.63 \times 10^{-8} \mathrm{~S} \mathrm{~cm}^{-1}$ at $353 \mathrm{~K}$ for 1 . As the temperature was increased, the electrical conductivity continuously increased, by a total of about three orders of magnitude to 4.35 $\times 10^{-5} \mathrm{~S} \mathrm{~cm}^{-1}$ for 1 at $413 \mathrm{~K}$. We found $\ln \sigma$ of 1 to be linearly related to $1 / T$ between $353 \mathrm{~K}$ and $413 \mathrm{~K}$ (Fig. 3c), and its activation energy $\left(E_{\mathrm{a}}\right)$ was estimated to be $25.8 \mathrm{kcal} \mathrm{mol}^{-1}$.

In summary, compound $\left[\mathrm{Cd}(\mathrm{en})_{3}\right] \mathrm{Ag}_{2} \mathrm{I}_{4}$ (1) was successfully prepared by using a simple self-assembly method. Compound 1 represents a rare noncentrosymmetric 3D framework hybrid iodoargentate with a metal complex as a template. Furthermore, 1 showed relaxor-like dielectric behavior and switchable dielectric properties in the investigated temperature range, and dielectric relaxation behavior was associated with local polarization effects due to the flexibility of the channel structure and small local movement of the Ag and I ions in the framework to create local dipole moments. These results revealed hybrid iodoargentates to have interesting relaxor-like properties and to be of potential use as switchable dielectric materials.

\section{Conflicts of interest}

There are no conflicts to declare.

\section{Acknowledgements}

The authors thank the National Nature Science Foundation of China, Natural Science Foundation of Jiangsu Province and Natural Science Training Foundation of Nanjing Xiaozhuang University for financial support (grant No: 21201103, 21301093, BK20171125 and 2016NXY12).

\section{Notes and references}

1 (a) S. Mishra, E. Jeanneau, G. Ledoux and S. Daniele, Inorg. Chem., 2014, 53, 11721-11731; (b) H. H. Li, Y. Y. Xing, Z. X. Lian, A. W. Gong, H. Y. Wu, Y. Li and Z. R. Chen, CrystEngComm, 2013, 15, 1721-1728; (c) T. Yu, Y. B. Fu, Y. L. Wang, P. F. Hao, J. J. Shen and Y. L. Fu, CrystEngComm, 2013, 17, 8752-8761.

2 (a) J. J. Shen, C. F. Zhang, T. L. Yu, L. An and Y. L. Fu, Cryst. Growth Des., 2014, 14, 6337-6342; (b) T. L. Yu, J. J. Shen, Y. L. Wang and Y. L. Fu, Eur. J. Inorg. Chem., 2015, 2015, 1989-1996.

3 (a) Z. Wang, G. L. Zhuang, Y. K. Deng, Z. Y. Feng, Z. Z. Cao, M. Kurmoo, C. H. Tung and D. Sun, Inorg. Chem., 2016, 55, 4757-4763; (b) Z. H. Yan, X. Y. Li, L. W. Liu, S. Q. Yu, X. P. Wang and D. Sun, Inorg. Chem., 2016, 55, 1096-1101; (c) X. W. Lei, C. Y. Yue, J. Zhang, Y. F. Han, J. T. Yang, R. R. Meng, C. S. Gao, H. Ding, C. Y. Wang, W. D. Chen and M. C. Hong, Inorg. Chem., 2015, 54, 10593-10603.

4 T. Devic, M. Evain, Y. Moelo, E. Canadell, P. Auban-Senzier, M. Fourmigué and P. Batail, J. Am. Chem. Soc., 2003, 125, 3295-3301.
5 (a) S. Mishra, E. Jeanneau, G. Ledoux and S. Daniele, Inorg. Chem., 2014, 53, 11721-11731; (b) H. H. Li, Y. Y. Xing, Z. X. Lian, A. W. Gong, H. Y. Wu, Y. Li and Z. R. Chen, CrystEngComm, 2013, 15, 1721-1728.

6 (a) W. X. Xu, W. X. Zhou, J. Li, S. L. Huang, J. Niu and F. X. Zhang, Inorg. Chem. Commun., 2014, 40, 220-222; (b) T. L. Yu, J. J. Shen, Y. B. Fu and Y. L. Fu, CrystEngComm, 2014, 16, 5280-5289.

7 (a) G. N. Liu, L. L. Liu, Y. N. Chu, Y. Q. Sun, Z. W. Zhang and C. C. Li, Eur. J. Inorg. Chem., 2015, 2015, 478-487; (b) L. Li, H. Chen, Y. Z. Qiao and Y. Y. Niu, Inorg. Chim. Acta, 2014, 409, 227-232; (c) W. Fang, C. Y. Tang, R. H. Chen, D. X. Jia, W. Q. Jiang and Y. Zhang, Dalton Trans., 2013, 42, 1515015158.

8 R. C. Zhang, Y. J. Zhang, B. Q. Yuan, J. P. Miao, B. H. Pei, P. P. Liu, J. J. Wang and D. J. Zhang, J. Solid State Chem., 2014, 220, 185-190.

9 (a) T. L. Yu, L. An, L. Zhang, J. J. Shen, Y. B. Fu and Y. L. Fu, Cryst. Growth Des., 2014, 14, 3875-3879; (b) X. W. Lei, C. Y. Yue, L. J. Feng, Y. F. Han, R. R. Meng, J. T. Yang, H. Ding, C. S. Gao and C. Y. Wang, CrystEngComm, 2016, 18, 427-436; (c) E. Cariati, R. Macchi, D. Roberto, R. Ugo, N. Casati, P. Macchi, A. Sironi, L. Bogani, A. Caneschi and D. Gatteschi, J. Am. Chem. Soc., 2007, 129, 9410-9420.

10 Y. S. Jiang, H. G. Yao, S. H. Ji, M. Ji and Y. L. An, Inorg. Chem., 2008, 47, 3922-3924.

11 (a) A. M. Guloy, Z. J. Tang, P. B. Miranda and V. I. Srdanov, Adv. Mater., 2001, 13, 833-837; (b) T. P. Radhakrishnan, Acc. Chem. Res., 2008, 41, 367-376; (c) G. R. Desiraju, Angew. Chem., Int. Ed., 2007, 46, 8342-8356.

12 H. B. Duan, H. R. Zhao, X. M. Ren, H. Zhou, Z. F. Tian and W. Q. Jin, Dalton Trans., 2011, 40, 1672-1683.

13 Y. B. Tong, L. T. Ren, H. B. Duan, J. L. Liu and X. M. Ren, Dalton Trans., 2015, 44, 17850-17858.

14 H. B. Duan, S. S. Yu, Y. B. Tong, H. Zhou and X. M. Ren, Dalton Trans., 2016, 45, 4810-4818.

15 (a) A. J. Neukirch, W. Nie, J. C. Blancon, K. Appavoo, H. Tsai, M. Y. Sfeir, C. Katan, L. Pedesseau, J. Even, J. J. Crochet, G. Gupta, A. D. Mohite and S. Tretiak, Nano Lett., 2016, 16, 3809-3816; (b) O. Yaffe, Y. S. Guo, L. Z. Tan, D. A. Egger, T. Hull, C. C. Stoumpos, F. Zheng, T. F. Heinz, L. Kronik, M. G. Kanatzidis, J. S. Owen, A. M. Rappe, M. A. Pimenta and L. E. Brus, Phys. Rev. Lett., 2017, 118, 136001-1136001-6.

16 (a) D. W. Fu, W. Zhang, H. L. Cai, Y. Zhang, J. Z. Ge, R. G. Xiong, S. P. Huang and T. Nakamura, Angew. Chem., Int. Ed., 2011, 50, 11947-11951; (b) Z. H. Sun, T. L. Chen, J. H. Luo and M. C. Hong, Angew. Chem., Int. Ed., 2012, 51, 3871-3876.

17 (a) J. C. Papaioannou and T. C. Ghikas, Mol. Phys., 2003, 101, 260-2608; (b) T. C. Ghikas and J. C. Papaioannou, Mol. Phys., 2002, 100, 673-679; (c) C. Ang and Z. Yu, Phys. Rev. B: Condens. Matter Mater. Phys., 2000, 61, 3922. 